\title{
Vertex-colouring edge-weightings with two edge weights
}

\author{
Mahdad Khatirinejad ${ }^{1 *}$ \\ Reza Naserasr ${ }^{2} \quad$ Mike Newman $^{3 *}$ \\ Ben Seamone $^{2 \dagger}$ \\ Brett Stevens ${ }^{2 *}$ \\ ${ }^{1}$ Department of Communications and Networking, Aalto University, Finland \\ ${ }^{2}$ School of Mathematics and Statistics, Carleton University, Canada \\ ${ }^{3}$ Department of Mathematics, University of Ottawa, Canada
}

received $24^{\text {th }}$ July 2010, accepted $24^{\text {th }}$ November 2011.

An edge-weighting vertex colouring of a graph is an edge-weight assignment such that the accumulated weights at the vertices yields a proper vertex colouring. If such an assignment from a set $S$ exists, we say the graph is $S$-weight colourable. It is conjectured that every graph with no isolated edge is $\{1,2,3\}$-weight colourable.

We explore the problem of classifying those graphs which are $\{1,2\}$-weight colourable. We establish that a number of classes of graphs are $S$-weight colourable for much more general sets $S$ of size 2 . In particular, we show that any graph having only cycles of length $0 \bmod 4$ is $S$-weight colourable for most sets $S$ of size 2 . As a consequence, we classify the minimal graphs which are not $\{1,2\}$-weight colourable with respect to subgraph containment. We also demonstrate techniques for constructing graphs which are not $\{1,2\}$-weight colourable.

Keywords: edge weighting, graph colouring

\section{Introduction}

Let $G$ be a simple graph and $S$ be a set of real numbers. An $S$-edge-weighting of $G$ is an assignment $w: E(G) \rightarrow S$. Given an $S$-edge-weighting, the weighted degree of a vertex $v$, denoted $w(v)$, is the sum of weights of the edges incident with $v$. An $S$-edge-weighting gives a vertex colouring if the weighted degrees of adjacent vertices are different. If an $S$-edge-weighting vertex colouring $w$ exists, we also call $w$ an $S$-weight colouring and we say $G$ is $S$-weight colourable. For a positive integer $k$, we say $G$ has a $k$-weight colouring or $G$ is $k$-weight colourable if it is $S$-weight colourable for every set $S$ of size $k$. The most commonly studied sets $S$ are those of the form $\{1, \ldots, k\}$.

Problem 1 Given a graph $G$ with no isolated edges, find the minimum $k$ such that $G$ is $\{1, \ldots, k\}$-weight colourable.

\footnotetext{
* Partially supported by NSERC Canada.

† Partially supported by Fonds québécois de la recherche sur la nature et les technologies.

‡ Emails: mahdad.khatirinejad@tkk.fi, \{bseamone, brett\}@math. carleton. ca, mnewman@uottawa.ca 
It is not hard to verify that $K_{4}$ with a single leaf attached is $\{1,2\}$-weight colourable but is not $\{0,1\}$ weight colourable. It follows that the $S$-weight colourability of a graph is not only dependent on the size of $S$ but also on the particular elements of $S$. However, if a graph $G$ is $S$-weight colourable then there exists an $i_{0}=i_{0}(G, S)$ such that for all $i>i_{0}$ the graph is also $\{s+i: s \in S\}$-weight colourable. One such value for $i_{0}$, though not necessarily the smallest, is $i_{0}=|S| \cdot \Delta(G) \cdot \max \{|s|: s \in S\}$, where $\Delta(G)$ is the maximum degree of $G$.

Let us start by considering the 2-weight colourability of a simple class of graphs - paths. If $a$ and $b$ are non-zero real numbers, then every path of length at least 2 has an $\{a, b\}$-weight colouring. Assigning the edge weights $a, a, b, b, a, a, b, b, \ldots$, beginning with one leaf of the path, gives such a colouring. However, a path has a $\{0, a\}$-weight colouring if and only if it is not of length $1 \bmod 4$. The reader can easily check that paths of length 2, 3 and 4 have a $\{0, a\}$-weight colouring. However, if we let $P=e_{1}, e_{2}, e_{3}, e_{4}, e_{5}$ be a path of length 5 (we omit vertex labels) then if $w\left(e_{2}\right)=0$ (or $w\left(e_{4}\right)=0$ ) then the ends of $e_{1}\left(e_{5}\right)$ will have equal weight. Thus the only way to achieve a $\{0, a\}$-weight colouring of $P$ is if $w\left(e_{2}\right)=w\left(e_{4}\right)=a$. However, this implies that the ends of $e_{3}$ will have the same weight, and hence a $\{0, a\}$-weight colouring cannot exist. These examples easily extend to longer paths; the details are left to the reader.

In general, it is unknown how difficult it is to decide if a given graph admits a $\{1,2\}$-weight colouring, or more generally an $\{a, b\}$-weight colouring. As such, we present the following question:

Problem 2 Is it NP-complete to decide whether a given graph is 2-weight colourable?

Returning to Problem 1. we state the following conjecture, due to Karoński, Łuczak, and Thomason [KŁT04], which motivates most of the known results on the $\{1, \ldots, k\}$-weight colourability of graphs.

Conjecture 1.1 Every graph with no isolated edge is $\{1,2,3\}$-weight colourable.

Karoński et al. [KŁT04] showed that the Conjecture 1.1 is true for 3-colourable graphs. They also proved that if $S$ is any set of at least 183 real numbers which are linearly independent over the rational numbers then every graph with no isolated edge is $S$-weight colourable. Recently, Kalkowski et al. [KKP09] showed that every graph with no isolated edge is $\{1, \ldots, k\}$-weight colourable for $k=5$. This result is an improvement on the previous bounds on $k$ established by Addario-Berry et al. [ABDM ${ }^{+} 07$ ], Addario-Berry et al. [ABDR08], and Wang et al. [WY08], who obtained the bounds $k=30, k=16$, and $k=13$, respectively.

Our work in this paper is similarly motivated by Conjecture 1.1. However, where most others have attempted to lower the best known value of $k$ as described above, our focus is on establishing which graphs are $\{1,2\}$-weight colourable. Addario-Berry, Dalal and Reed ABDR08 showed that asymptotically almost every graph is $\{1,2\}$-weight colourable, however it is not known which ones are not. Chang et al and Lu et al ([CLWY10], [LYZ10]) have made some progress in determining which classes of graphs are $\{1,2\}$-weight colourable, notably having shown that 3 -connected bipartite graphs are one such class. A complete classification of such graphs would determine those graphs for which $k=3$ is the smallest possible solution in Problem 1 , and would reduce Conjecture 1.1 to just those graphs.

The results that follow are, for the most part, concerned with a more general problem than that of finding $\{1,2\}$-weight colourings, namely that of finding $\{a, b\}$-weight colourings for more general values of $a$ and $b$. In such cases, the existence of a $\{1,2\}$-weight colouring follows as an unstated corollary. In Section 2 . we establish a wide range of basic graphs which admit $\{a, b\}$-weight colourings. We also establish classes of graphs which do not admit $\{a, b\}$-weight colourings, but which do admit an $\{a, b\}$-edge weighting which is almost a proper colouring. These results provide building blocks for our results on the weight 
colourability of bipartite graphs in Section 3 and of other general classes of graphs, particularly direct products of graphs, in Section 4 . Of note, we show in Section 3 that if every cycle of $G$ is of length $0 \bmod 4$, then $G$ is $\{1,2\}$-weight colourable.

\section{Building blocks: Weight colourings of basic graphs}

We will use standard graph theory terminology; the reader may refer to [BM08] for clarification of any terms which are not specifically defined here.

The length of a path (walk) is defined to be the number of edges of the path (walk). A thread in a graph $G$ is a walk connecting two vertices $x$ and $y$, not necessarily distinct, such that the internal vertices are distinct from all others on the walk, all internal vertices have degree 2 in $G$, and $\operatorname{deg}(x), \operatorname{deg}(y) \geq 3$. If $x$ and $y$ are distinct, then the walk is in fact a path and in this case we may refer to the thread as an ear. If the condition that $\operatorname{deg}(x), \operatorname{deg}(y) \geq 3$ is changed to $\operatorname{deg}(x), \operatorname{deg}(y) \geq 2$ in either case, we have a subthread or subear respectively.

A cut vertex of a graph is one whose removal disconnects the graph. A graph is 2-connected if it has no cut vertex. A graph (not necessarily simple) is called separable if it can be decomposed into two nonempty subgraphs with exactly one vertex in common. A simple graph is separable if and only if it is not 2-connected. A maximal nonseparable subgraph of $G$ is a block of $G$. Note that a block is isomorphic either to $K_{2}$ or to a 2-connected graph. An end block of $G$ is a block which contains at most one cut vertex of $G$.

A graph is c-colourable if the vertices can be coloured with $c$ colours so that adjacent vertices get different colours.

$K_{n}$ and $C_{n}$, respectively, denote the complete graph and the cycle on $n$ vertices. The Cartesian product of two graphs $G$ and $H$, denoted by $G \square H$, is defined as the graph having vertex set $V(G) \times V(H)$ where two vertices $\left(u, u^{\prime}\right)$ and $\left(v, v^{\prime}\right)$ are adjacent if and only if either $u=v$ and $u^{\prime}$ is adjacent to $v^{\prime}$ in $H$ or $u^{\prime}=v^{\prime}$ and $u$ is adjacent to $v$ in $G$.

We present a few simple observations.

Proposition 2.1 Let $a, b, t$ be nonzero real numbers and $G$ a graph. Then

(i) $G$ is $\{a, b\}$-weight colourable if and only if $G$ is $\{a t, b t\}$-weight colourable, and

(ii) if $G$ is $\{a, b\}$-weight colourable then $G$ is $\{p, q\}$-weight colourable for any nonzero $p, q \in \mathbb{R}$ which are linearly independent over $\mathbb{Q}$.

Proof: (i) This follows from the fact that $w(u) \neq w(v)$ if and only if $t \cdot w(u) \neq t \cdot w(v)$. (ii) Note that if two adjacent vertices receive distinct linear combinations of $a$ and $b$ as weights, then the coefficients of these linear combinations will suffice for any two linearly independent nonzero reals.

From Proposition 2.1 we deduce the following, adopting the convention that 0 and 1 are relatively prime integers:

Corollary 2.2 A graph $G$ is 2-weight colourable if and only if $G$ is $\{a, b\}$-weight colourable for every pair of relatively prime integers $a$ and $b$.

Proposition 2.1 allows us to reduce our proofs of positive results on the existence of $\{a, b\}$-weight colourings of a graph to relatively prime integers. Results in which we show that $G$ does not admit an $\{a, b\}$-weight colouring will not rely on such assumptions - we will prove them for all real $a, b$. 
Proposition 2.3 If $G$ is $d$-regular and $\{a, b\}$-weight colourable for a fixed choice of $a$ and $b$ then $(i)$ it is $d$-colourable, and (ii) it is 2-weight colourable.

Proof: (i) The weighted degree of each vertex must be a number of the form $t a+(d-t) b$ for some $0 \leq t \leq d$, and a vertex of weighted degree $d a$ cannot be adjacent to a vertex of weighted degree $d b$. Thus putting the vertices of weighted degree $d a$ or $d b$ in the same colour class gives a $d$-colouring.

(ii) In an $\{a, b\}$-edge weighting of a $d$-regular graph, the accumulated weight at any vertex is in a oneto-one correspondence with the number of incident edges of weight $a$. Thus if one choice of $a$ and $b$ gives a vertex colouring, then any other choice of $a$ and $b$ will as well.

Corollary 2.4 If $\chi(G)=\Delta(G)+1$ or, equivalently (by Brooks theorem), if $G$ is an odd cycle or a complete graph then $G$ is not $S$-weight colourable for any set $S$ of size 2 .

Even though the complete graph is not $S$-weight colourable for any set of size 2, it has an $S$-edgeweighting that is very close to being an $S$-weight colouring. This specific weighting will be useful in constructing families of 2-weight colourable graphs and non-2-weight colourable graphs in Section 4.

Lemma 2.5 Given $n \geq 2$ and $a \neq b \in \mathbb{R}$, there is an $\{a, b\}$-edge-weighting of $K_{n}$ such that the weighted degrees of all the vertices are distinct except for 2 of them. Furthermore, in any such $\{a, b\}$ edge-weighting, the degree sequence of the subgraph induced by the edges of weight a (as well as the subgraph induced by the edges of weight b) is either

$$
\left(1,2, \ldots,\left\lfloor\frac{n}{2}\right\rfloor-1,\left\lfloor\frac{n}{2}\right\rfloor,\left\lfloor\frac{n}{2}\right\rfloor,\left\lfloor\frac{n}{2}\right\rfloor+1, \ldots, n-2, n-1\right),
$$

or

$$
\left(0,1, \ldots,\left\lceil\frac{n}{2}\right\rceil-2,\left\lceil\frac{n}{2}\right\rceil-1,\left\lceil\frac{n}{2}\right\rceil-1,\left\lceil\frac{n}{2}\right\rceil, \ldots, n-3, n-2\right) .
$$

Proof: We prove the first part with an explicit construction. Choose any two vertices and assign weight $a$ to the edge joining them. Choose a new vertex and assign weight $b$ to all the edges joining this vertex to the previous two vertices. Choose another vertex and assign weight $a$ to all the edges joining this vertex to the previous three vertices. By repeating this process until all vertices are exhausted, we achieve the desired edge-weighting since the two vertices chosen first will have the same weight while the remainder of the graph is properly coloured. Note that we achieve the same result by swapping $a$ and $b$ in this argument.

We prove the second part of the lemma by induction on $n$. Suppose $w$ is such an edge-weighting of $K_{n}$ and let $w(u)=w(v)$. It is easy to verify the claim for $n=2$ and $n=3$. If $w(x) \notin\{(n-1) a,(n-1) b\}$ for every vertex $x$ then $w(x)$ can only take $n-2$ values, a contradiction to the choice of $w$. If $w(u)=$ $w(v) \in\{(n-1) a,(n-1) b\}$ then by removing $u$ and $v, w$ induces an $\{a, b\}$-weight colouring of $K_{n-2}$, a contradiction to Corollary 2.4 Thus there exists a vertex $x \neq u, v$ such that $w(x) \in\{(n-1) a,(n-1) b\}$. The claim follows by induction on $K_{n}-x$.

The following technical lemmata will be useful for the rest of the paper, since they establish useful tools for finding edge-weighting vertex colourings of graphs with specific structural properties.

Lemma 2.6 Suppose $G$ has a vertex $v$ with a set of leaf neighbours $L$ where $|L| \geq\lceil\operatorname{deg}(v) / 2\rceil$. Let $a \neq b$ be real numbers with $a b>0$. If $G \backslash L$ is $\{a, b\}$-weight colourable, then so is $G$. 
Proof: As mentioned, Proposition 2.1 allows us to only consider $a, b \in \mathbb{Z}^{+}$. Suppose $w$ is an $\{a, b\}-$ weight colouring of $G \backslash L$. The possible extensions of $w$ to $G$ give exactly $|L|+1$ possible weights for $v$. Since $v$ has at most $|L|$ neighbours in $G \backslash L$, in at least one of the extensions, the weighted degree of $v$ is different from the weighted degrees of the neighbours of $v$ in $G \backslash L$. The weighted degree of $v$ is also different from the weighted degrees of the neighbours of $v$ in $L$, since $a b>0$.

Corollary 2.7 Every tree with at least 3 vertices is $\{a, b\}$-weight colourable, where $a \neq b$ are real numbers with $a b>0$.

Proof: The statement holds for any star, $K_{1, n-1}$, since the assignment of $a$ to all edges achieves the desired result. As such the result holds for $n=3$ since the unique tree on 3 vertices is a star. Let $T$ be a tree on $n$ vertices which is not a star and assume the result holds for any tree with fewer than $n$ vertices. Every tree has a vertex $v$ that has at least $\lceil\operatorname{deg}(v) / 2\rceil$ leaf neighbours. Since $T$ is not a star, removing the leaf neighbours of $v$ gives a subtree $T^{\prime}$ on at least 3 vertices. By the induction hypothesis $T^{\prime}$ has an $\{a, b\}$-weight colouring. By Lemma 2.6 $T$ does as well.

The following lemma establishes that we may contract long threads in a way that maintains weight colourability.

Lemma 2.8 Let $G$ be a graph, $P=v_{0}, e_{1}, v_{1}, e_{2}, v_{2}, e_{3}, v_{3}, e_{4}, v_{4}, e_{5}, v_{5}$ be a subthread of $G$, and $a \neq b$ be any two real numbers. Let $G^{\prime}=G /\left\{e_{1}, e_{2}, e_{3}, e_{4}\right\}$ Then,

(i) If $w$ is an $\{a, b\}$-weight colouring of $G$, then $w\left(e_{1}\right)=w\left(e_{5}\right) \neq w\left(e_{3}\right)$.

(ii) If $G^{\prime}$ is $\{a, b\}$-weight colourable, then so is $G$.

(iii) If $\operatorname{deg}\left(v_{0}\right)=2$ or $\operatorname{deg}\left(v_{5}\right)=2$, then $G$ is $\{a, b\}$-weight colourable if and only if $G^{\prime}$ is $\{a, b\}$-weight colourable.

Proof: (i) If $w\left(e_{1}\right) \neq w\left(e_{5}\right)$ then either one of the two choices for $w\left(e_{3}\right)$ results in an improper colouring at $e_{2}$ or $e_{4}$. Hence $w\left(e_{1}\right)=w\left(e_{5}\right)$ and $w\left(e_{3}\right)$ must be distinct.

(ii) For convenience, we still denote the vertex obtained from the contraction by $v_{0}$. Suppose $w^{\prime}$ is an $\{a, b\}$-weight colouring of $G^{\prime}$. Then $w^{\prime}\left(v_{0}\right) \neq w^{\prime}\left(v_{5}\right)$. Without loss of generality assume $w^{\prime}\left(v_{0} v_{5}\right)=a$. Let $w(e)=w^{\prime}(e)$ for each $e \notin\left\{e_{1}, e_{2}, e_{3}, e_{4}, e_{5}\right\}, w\left(e_{1}\right)=w\left(e_{5}\right)=a$ and $w\left(e_{3}\right)=b$. There are two possibilities for the weights of $e_{2}$ and $e_{4}$. Assigning $w\left(e_{2}\right)=a$ and $w\left(e_{4}\right)=b$ does not yield a proper vertex colouring of $G$ if and only if either $w\left(v_{0}\right)=2 a$ or $w\left(v_{5}\right)=a+b$. Similarly, defining $w\left(e_{2}\right)=b$ and $w\left(e_{4}\right)=a$ does not yield a proper vertex colouring of $G$ if and only if either $w\left(v_{0}\right)=a+b$ or $w\left(v_{5}\right)=2 a$. Suppose that neither weighting works. If the first possibility gives $w\left(v_{0}\right)=2 a$, then the second must give $w\left(v_{5}\right)=2 a$. If the first possibility gives $w\left(v_{5}\right)=a+b$, then the second gives $w\left(v_{0}\right)=a+b$. In either case $w\left(v_{0}\right)=w\left(v_{5}\right)$, a contradiction.

(iii) Assume $\operatorname{deg}\left(v_{0}\right)=2$ and let $e_{0}$ be the other edge incident with $v_{0}$. Suppose $w$ is an $\{a, b\}$-weight colouring of $G$. By (i) we have $w\left(e_{0}\right)=w\left(e_{4}\right)$ and $w\left(e_{1}\right)=w\left(e_{5}\right)$. Hence $w\left(v_{0}\right)=w\left(v_{4}\right) \neq w\left(v_{5}\right)$. Thus, by assigning the common weight of $e_{1}$ and $e_{5}$ to the edge $v_{0} v_{5}$, we get an $\{a, b\}$-weight colouring of $G^{\prime}$.

The degree condition on the ends of $P$ in Lemma 2.8(iii) cannot be dropped. For example, by taking $G$ to be the path of length $5, a=1$, and $b=2$, Lemma 2.8 (iii) fails. 
From this lemma we may deduce necessary and sufficient conditions for the existence of $\{a, b\}$-weight colourings of cycles.

Proposition 2.9 Let $a$ and $b$ be any distinct real numbers. Then $C_{n}$ is $\{a, b\}$-weight colourable if and only if $n \equiv 0(\bmod 4)$.

In lieu of a proof, we simply note that, by Lemma2.8(iii), the proof of this proposition may be reduced to the cases $C_{3}, C_{4}, C_{5}$ and $C_{6}$. The details are left to the reader. There are $\{a, b\}$-edge weightings of other cycles of length $4 k+1,4 k+2$ and $4 k+3$ which give vertex colourings with as few conflicts as possible. These results are largely technical, though not difficult to prove.

Proposition 2.10 Let $a$ and $b$ be any distinct real numbers. Then $C_{2 k+1}$ has an $\{a, b\}$-edge weighting $w$ such that only one edge $e=u v$ has the property that $w(u)=w(v)$.

Proposition 2.11 Let $a$ and $b$ be any distinct real numbers. Then $C_{4 k+2}$ has an $\{a, b\}$-edge weighting $w$ such that precisely two edges $e=u v$ and $e^{\prime}=u^{\prime} v^{\prime}$ have the property that $w(u)=w(v)$ and $w\left(u^{\prime}\right)=$ $w\left(v^{\prime}\right)$. Furthermore,

- the distance between $e$ and $e^{\prime}$ is even,

- $e$ and $e^{\prime}$ may be chosen to be any two edges at an even distance, and

- if $f_{1}$ and $f_{2}$ are the edges incident to e, then their weights are equal and can be chosen to be either a or $b$ (similar for $e^{\prime}$ ).

We present a specific consequence of Proposition 2.11 which we will find useful.

Proposition 2.12 Let $k$ be an integer, $k \geq 1$. Then $C_{4 k+2}$ has an $\{a, b\}$-edge-weighting such that three consecutive vertices have equal weight and the rest of the cycle is properly coloured. Furthermore, the edge-weighting can be chosen so that the weights of the four edges which contribute to the weights of those three vertices will all be $a$, all $b$, or alternate between $a$ and $b$.

Let $\Theta_{\left(m_{1}, \ldots m_{d}\right)}, d \geq 3$, be the graph constructed from $d$ internally disjoint paths between distinct vertices $x$ and $y$, where the $i$-th path has of length $m_{i}$. For simplicity, we assume $m_{1} \leq m_{2} \leq \cdots \leq m_{d}$. Such graphs will be referred to as theta graphs. We present necessary and sufficient conditions for theta graphs to be 2-weight colourable.

Theorem 2.13 Let $d \geq 3$ and let $a, b$ be real numbers. The graph $\Theta_{\left(m_{1}, m_{2}, \ldots, m_{d}\right)}$ is 2-weight colourable if and only if it is not of the form $\Theta_{\left(1,4 k_{2}+1, \ldots, 4 k_{d}+1\right)}$.

Proof: Let $x$ and $y$ be the two vertices of degree greater than two, and let $\left\{P_{i} \mid 1 \leq i \leq d\right\}$ be the $d$ internally disjoint paths between $x$ and $y$.

Suppose $w$ is an $\{a, b\}$-weight colouring of $\Theta_{\left(1,4 k_{2}+1, \ldots, 4 k_{d}+1\right)}$. By applying Lemma 2.8 (i) to each $P_{i}$, we observe that on any of the $d$ disjoint paths between $x$ and $y$ the first and last edges must receive same weight. Thus $w(x)=w(y)$, a contradiction since $x$ and $y$ are adjacent. Hence $\Theta_{\left(1,4 k_{2}+1, \ldots, 4 k_{d}+1\right)}$ is not $\{a, b\}$-weight colourable for any $a, b$.

Consider $\Theta_{\left(m_{1}, m_{2}, \ldots, m_{d}\right)} \nsucceq \Theta_{\left(1,4 k_{2}+1, \ldots, 4 k_{d}+1\right)}$. We can assume that $|a| \geq|b|$. Let $n_{j}$ be the number of paths that have length equivalent to $j \bmod 4$. Note that $n_{0}+n_{1}+n_{2}+n_{3}=d=\operatorname{deg}(x)=\operatorname{deg}(y)$. For each path $P_{i}$, weight each edge according to Lemma 2.8 so that the edges incident with $x$ are weighted 
$a$. Then $w(x)=d a$ so it has no conflicts with its neighbours since $d \geq 3$ and the condition on the magnitudes of $a$ and $b$ gives $d a \notin\{2 a, a+b\}$. Note that, if $\left|P_{i}\right| \geq 2$, there are two choices for the next edge's weight on $P_{i}$ which determines the rest of the weights. Given one such weighting of a path $P_{i}$, the effects of switching to the alternate weighting where the edge incident to $x$ receives weight $a$ depend on the parity of the length of the path. If $\left|P_{i}\right|$ is even, the weight of the edge incident to $y$ and the vertex weights of the neighbours of $x$ and $y$ on $P_{i}$ all change. If $\left|P_{i}\right|$ odd, the weight of the edge incident to $y$ remains unchanged, but the vertex weights of the neighbours of $x$ and $y$ on $P_{i}$ do change. In all cases the only possible weights on path-neighbours of $x$ or $y$ are $2 b, a+b$ and $2 b$. We prove, by cases, that there is an appropriate set of choices which make $w(y)$ distinct from its neighbours.

$\mathbf{n}_{\mathbf{0}}+\mathbf{n}_{\mathbf{2}} \geq 4$ : Our choice of weightings for even $P_{i}$ 's give at least 5 possible values for $w(y)$, so there is a choice such that $w(y) \notin\{2 b, a+b, 2 a, d a\}$.

$\mathbf{n}_{\mathbf{0}}+\mathbf{n}_{\mathbf{2}}=3$ : If no $P_{i}$ has length $1, d a$ is not a forbidden weight for $y$. Also, if $n_{3} \geq 1$ then there is an edge incident to $y$ with weight $b$, and $w(y) \neq d a$. In either case there is a choice of weightings so that $w(y) \notin\{2 b, a+b, 2 a\}$.

So, assume that $m_{1}=1$ and $n_{3}=0$. If the initial weighting fails then we must have

$$
\{2 b, a+b, 2 a, d a\}=\{(d-3) a+3 b,(d-2) a+2 b,(d-1) a+b, d a\}
$$

which implies that $b=-(d-3) a$ and $d \geq 4$. The fact that $|a| \geq|b|$ gives that $d=4$, implying $n_{1}=1$ and $b=-a$. We weight all edges explicitly. The single edge on the path of length 1 receives weight $a$. If $n_{0}=3$ then weight the edges of one even path $a, a, \ldots,-a,-a$ and the other two $a,-a, \ldots,-a, a$. If $n_{0}=2$ and $n_{2}=1$ weight the edges of the paths of length $0 \bmod 4$ with $a, a, \ldots,-a,-a$ and the other even path with $a, a, \ldots, a, a$. If $n_{0}=1$ and $n_{2}=2$ weight the edges of the path of length $0 \bmod 4$ with $a,-a, \ldots,-a, a$ and the two other even paths with $a,-a, \ldots, a,-a$. Finally if $n_{0}=0$ and $n_{2}=3$ weight the edges of all even paths with $a,-a, \ldots, a,-a$. Each weighting gives a vertex-colouring for its respective case.

$\mathbf{n}_{\mathbf{0}}+\mathbf{n}_{\mathbf{2}}=\mathbf{2}$ : If $n_{3}=0$ and $n_{0}>0$ then assign weights to the edges of one path which is length $0 \bmod 4$ so that the weights of the first and last edges are both $a$. Weight the edges of the other even path so that the edge incident to $x$ is weighted $a$ and the edge incident to $y$ is weighted $b$. If $n_{3}=n_{0}=0$ but either $d>3$ or $b \neq 0$ then assign weights to the edges of both even paths so that their edges incident with $x$ are weighted $a$, one of the edges incident with $y$ is weighted $a$ and the other is weighted $b$. In both cases weight the edges of the paths of length $1 \bmod 4$ so the weights are, in order beginning with the edge incident with $x, a, a \ldots b, a$ (if the path is a single edge, give it weight $a$ ). In the case when $n_{3}=n_{0}=0$, $d=3$ and $b=0$ weight the edges of the two even paths $a, 0,0, \cdots a, a, 0$ and the single odd path with $0,0, a, a, \cdots a, 0$ (beginning with the edge incident with $x$ in each case). The weighting given in each case gives a proper vertex colouring.

Assume $n_{3} \geq 1$. If $n_{0} \neq n_{2}$ then choose weightings for each $P_{i}$ so that $w(x)=d a$ and each remaining neighbour of $y$ has accumulated weight $a+b$. Then $w(y)=a n_{0}+a n_{1}+b n_{2}+b n_{3}$. Since $n_{3} \geq 1$ we have $w(x) \neq w(y)$, so the only possible conflict is if $w(y)=a+b$. In this case change both even $P_{i}$ 's to 
their alternate weighting, maintaining $w(x)=d a$ and producing a new weight at $y$ :

$$
\begin{aligned}
w^{\prime}(y) & =b n_{0}+a n_{1}+a n_{2}+b n_{3} \\
& =w(y)+(a-b)\left(n_{2}-n_{0}\right) \\
& =a+b+(a-b)\left(n_{2}-n_{0}\right) \\
& = \begin{cases}3 a-b & \text { if } n_{0}=0, n_{2}=2 \\
3 b-a & \text { if } n_{0}=2, n_{2}=0\end{cases}
\end{aligned}
$$

In either case $w^{\prime}(y) \neq a+b$. If $n_{0}=2$ then $y$ has neighbours with weights $2 b$, and $3 b-a \neq 2 b$. Similarly if $n_{2}=2$ then the weight at $y$ avoids conflict with its neighbours with weight $2 a$.

If $n_{0}=n_{2}=1$ we start again with choices from the basic strategy that leave all path-neighbours with weight $a+b$. We have $w(x)=d a \neq\left(n_{1}+1\right) a+\left(n_{3}+1\right) b=w(y)$. Thus the only conflict can again be if $w(y)=a+b$ or equivalently, $a n_{1}+b n_{3}=0$. In this case we weight the edges of $P_{i}$ 's of lengths equivalent to $0,1,2$, and $3 \bmod 4$ with $\{a, a, \ldots, b, b\},\{a, b, \ldots, a, a\},\{a, a, \ldots, a, a\}$ and $\{a, b, \ldots, b, b\}$ respectively. We still have that $w(y)=a+b \neq d a=w(x)$ and no neighbour of $y$ has weight $a+b$.

$\mathbf{n}_{\mathbf{0}}+\mathbf{n}_{\mathbf{2}}=1$ : If $n_{3}=0$ then weight the edges of the even path so that the edge incident with $x$ receives weight $a$ and the edge incident with $y$ receives weight $b$. Weight the edges of the paths of length $1 \bmod 4$ so the weights are, in order beginning with the edge incident with $x, a, a \ldots b, a$ (if the path is a single edge, give it weight $a$ ). This weighting gives a proper vertex colouring. Assume $n_{3} \geq 1$. Again, weight the edges of each $P_{i}$ so that $w(x)=d a$ and each neighbor of $y$ (distinct from $x$ ) has accumulated weight $a+b$. Since $n_{3} \geq 1$ we have that $w(x)=d a \neq w(y)$. If $w(y) \neq a+b$, then $w$ is an $\{a, b\}$-weight colouring. Suppose $w(y)=a+b$. Equivalently

$$
\left(n_{0}+n_{1}-1\right) a+\left(n_{2}+n_{3}-1\right) b=0 .
$$

Change the edge weights of the even length path to begin with $b, a$. Call this weighting $w^{\prime}$. We now have $w^{\prime}(x)=(d-1) a+b$ and $w^{\prime}(y) \neq a+b$. All neighbours of $y$ still have weight $a+b$, so the only possible conflicts are between $x$ and its neighbours. We reduce all potential conflicts to one of four cases, which are solved explicitly.

If $w^{\prime}(x)=w^{\prime}(y)$ then since $w^{\prime}(x)=(d-1) a+b, y$ is incident with precisely one edge with weight $b$. Since $n_{3} \geq 1$, the edge with weight $b$ comes from a path of length $3 \bmod 4$. This gives $n_{0}=0, n_{2}=1$ and $n_{3}=1$ and then Equation 1 and $|a| \geq|b|$ gives either

- $n_{0}=0, n_{1}=1, n_{2}=1, n_{3}=1$ and $b=0$ (case iii. below).

- $n_{0}=0, n_{1}=2, n_{2}=1, n_{3}=1$ and $b=-a$ (case iv. below).

The neighbours of $x$ have accumulated weights either $a+b$ or $2 a$. If $w(x)=(d-1) a+b=a+b$ then this implies that $d=2$ but the hypotheses of the theorem include $d \geq 3$. If $w(x)=(d-1) a+b=2 a$ then $b=-(d-3) a$. The fact that $d \geq 3$ and $|a| \geq|b|$ now give either

- $n_{0}=1, n_{1}=0, n_{2}=0, n_{3}=2$ and $b=0$ which is dealt with in case $\mathbf{i}$. below.

- $n_{0}=1, n_{1}=1, n_{2}=0, n_{3}=2$ and $b=-a$ which is dealt with in case ii. below.

- $n_{0}=0, n_{1}=1, n_{2}=1, n_{3}=1$ and $b=0$ which is dealt with in case iii. below. 
- $n_{0}=0, n_{1}=2, n_{2}=1, n_{3}=1$ and $b=-a$ which is dealt with in case iv. below.

case i. In this case $x$ and $y$ are not adjacent. Weight the edges of the path of length equivalent to $0 \bmod 4$ with $0,0, \ldots, a, a$ and the two odd paths with $a, 0, \ldots, 0,0$.

case ii. In this case $x$ and $y$ are not adjacent. Weight the edges of the paths of lengths equivalent to $0 \bmod$ 4, $1 \bmod 4$ and $3 \bmod 4$ with $-a,-a, \ldots, a, a, a,-a, \ldots, a, a$ and $a,-a, \ldots,-a,-a$ respectively.

case iii. In this case $x$ and $y$ may be adjacent. Weight the edges of the paths of lengths equivalent to $1 \bmod 4,2 \bmod 4$ and $3 \bmod 4$ with $a, a, \ldots, 0, a, 0,0, \ldots, 0,0$ and $0,0, \ldots, 0, a$ respectively.

case iv. In this case $x$ and $y$ may be adjacent. Weight the edges of the paths of lengths equivalent to $1 \mathrm{mod}$ 4, $2 \bmod 4$ and $3 \bmod 4$ with $a,-a, \ldots, a, a,-a,-a, \ldots,-a,-a$ and $a,-a, \ldots,-a,-a$ respectively.

Each of these edge-weightings gives a proper vertex colouring.

$\mathbf{n}_{\mathbf{0}}+\mathbf{n}_{\mathbf{2}}=\mathbf{0}$ : Every weighting of the paths $P_{i}$ which gives $w(x)=d a$ must give $w(y)=a n_{1}+b n_{3}$. If $m_{1}=1$ then, since our graph is not $\Theta_{\left(1,4 k_{2}+1, \ldots, 4 k_{d}+1\right)}$, we have $n_{3} \geq 1$ and thus $w(x) \neq w(y)$. Suppose $m \neq 1$. For each $P_{i}$ we have two choices for $y$ 's neighbour. Each choice leaves $w(y)$ constant. Thus there is a choice for each path which gives an edge-weighting vertex-colouring.

\section{Bipartite graphs}

We begin the section by noting that the property of being $\{a, b\}$-weight colourable is not one that is inherited by subgraphs, nor is the property of being non- $\{a, b\}$-weight colourable. For example, the graph consisting of $K_{4}$ with a leaf attached is $\{1,2\}$-weight colourable, however $K_{4}$ is not $\{a, b\}$-colourable for any choice of $a$ and $b$. Similarly $K_{4}$ contains the subgraph $C_{4}$ which is 2 -weight colourable.

We can, however, characterize the minimal graphs with respect to subgraph containment in the class of graph which are not $\{a, b\}$-weight colourable for many pairs $\{a, b\}$ (in particular, $\{1,2\}$ ). In Theorem 3.9 we establish that any graph which is not $\{a, b\}$-weight colourable must contain $C_{2 k+1}$ or $C_{4 k+2}$ as a subgraph for some positive integer $k$.

Definition 3.1 A graph $G$ is round if every cycle of $G$ has length $0 \bmod 4$.

The class of round graphs is much richer than merely those obtained by taking a graph and subdividing each edge into a path of length 4 . For example, $\Theta_{(2,2,2)} \cong K_{2,3}$ is a round graph which is not obtained in this way.

The following lemma establishes a useful subgraph condition of round graphs which we will use in our study of the $\{a, b\}$-weight colourability of round graphs.

Proposition 3.2 If $G$ is a round graph and $\Theta_{(i, j, k)}$ is a subgraph of $G$, then $i, j$ and $k$ are even and $i \equiv j \equiv k(\bmod 4)$.

Proof: Let $\Theta_{(i, j, k)}$ be a subgraph of $G$ and let $P_{i}, P_{j}$ and $P_{k}$ be the corresponding paths of length $i, j$ and $k$ respectively. Since $G$ is round, $i+j \equiv i+k \equiv j+k \equiv 0(\bmod 4)$. The result follows.

Before proceeding with our results on bipartite graphs we present the following definition which we adopt throughout this section (and this section only). In any $\{a, b\}$-edge-weighting of a graph, the weighted degree of every vertex is of the form $r a+s b$ for some nonnegative integers $r, s$. We will call a weighted vertex even (odd) if its weighted degree is $r a+s b$ with $r$ even (odd). Note that the parity 
of a weighted vertex does not necessarily refer to the parity of its weight. However, by Proposition 2.1. if $a$ and $b$ are not independent over $\mathbb{Q}$ then we will assume that they are relatively prime integers, and so we will assume that $a$ is an odd integer in this case. If $b$ is even, which will be the case in a number of the following results, then the parity of a weighted vertex does coincide with the parity of its weight.

Since a number of our arguments rely on this notion of parity, we often exclude those pairs of numbers whose ratio may be reduced to a ratio of odd integers. We define the sets:

$$
\begin{aligned}
& \mathcal{E}=\left\{\begin{array}{l|l|l}
\{a, b\} & \left.\frac{a}{b}=\frac{p}{q}, p, q \text { odd integers }\right\}
\end{array}\right. \\
& \mathcal{N}=\left\{\begin{array}{l|l}
\{a, b\} & \frac{a}{b}=\frac{p}{q}, p, q \in \mathbb{Z}, p q \leq 0
\end{array}\right\}
\end{aligned}
$$

We have already seen examples of bipartite graphs which are 2-weight colourable $\left(C_{4 k}\right.$ for any $k \geq 1$, bipartite theta graphs except $\left.\Theta_{\left(1,4 k_{2}+1, \ldots, 4 k_{d}+1\right)}\right)$ and some which are not $\left(C_{4 k+2}\right.$ for any $\left.k \geq 1\right)$. From these examples, we note that a bipartite graph $G$ with both parts of odd size is not necessarily $\{a, b\}$ weight colourable. However, if $G$ has one part of even size, we are able to prove $G$ is $\{a, b\}$-weight colourable for particular values of $a$ and $b$.

Theorem 3.3 Let $a, b \in \mathbb{R}$ be such that $\{a, b\} \notin \mathcal{E}$. If $G$ is a connected bipartite graph with at least one part being of even size, then $G$ is $\{a, b\}$-weight colourable.

Proof: Let $V(G)=X \cup Y$ be a bipartition of the vertices of $G$ with $|X|$ even. By Corollary 2.2 and since $\{a, b\} \notin \mathcal{E}$, we may assume that $a$ is an odd integer and $b$ is an even integer. We assign the weight $b$ to each edge of $G$. Clearly $v$ is even for each $v \in V(G)$. Let $V(X)=\left\{x_{1}, x_{2} \ldots x_{2 k}\right\}$ and let $P_{i}$ be an $x_{2 i-1} x_{2 i}$-path in $G$. By changing every edge weight along $P_{1}$ we only change the parity of $x_{1}$ and $x_{2}$. By repeating this process for each $P_{i}$ we have that every vertex of $X$ has odd parity and every vertex of $Y$ has even parity.

Call the resulting edge-weighting $w$. Suppose that $w$ is not an $\{a, b\}$-weight colouring. Then there are adjacent vertices $x$ and $y$ such that $w(x)=w(y)$. Thus there exist integers $r, r^{\prime}, s, s^{\prime}$ such $w(x)=r a+s b$ where $r$ is odd, $w(y)=r^{\prime} a+s^{\prime} b$ where $r^{\prime}$ is even, and $r a+s b=r^{\prime} a+s^{\prime} b$. If $a$ and $b$ are linearly independent over $\mathbb{Q}$, we must have $r=r^{\prime}$, a contradiction. Hence $b=(p / q) a$ for some $p, q \in \mathbb{Z}$ with $\operatorname{gcd}(p, q)=1$. Thus $r q+s p=r^{\prime} q+s^{\prime} p$. Since $r$ is odd and $r^{\prime}$ is even, $p$ even implies $q$ must be even, a contradiction. Hence $p$ is odd. Similarly, $q$ is odd. Therefore, $b / a=p / q$ with $p, q$ odd, contradicting our choice of $a$ and $b$. Thus $w$ is an $\{a, b\}$-weight colouring of $G$.

Corollary 3.4 Let $a, b \in \mathbb{R}$ be such that $\{a, b\} \notin \mathcal{E} \cup \mathcal{N}$. Let $G \neq K_{2}$ be a connected bipartite graph with a vertex of degree 1 . Then $G$ is $\{a, b\}$-weight colourable. In particular, trees are $\{a, b\}$-weight colourable.

Proof: Let $V(G)=X \cup Y$ be a bipartition of the vertices of $G$. Let $x \in X$ be a vertex of degree 1 and let $y \in Y$ be its neighbour. If $|X|$ or $|Y|$ is even, then $G$ is $\{a, b\}$-weight colourable by Theorem 3.3 . If $|X|$ is odd, then $G-x$ has an $\{a, b\}$-weight colouring by Theorem 3.3, say $w^{\prime}$, such that vertices in $X \backslash\{x\}$ are odd and vertices in $Y$ are even. By assigning $b$ to the edge $x y$ we maintain the parity of all the vertices. Also, since $\{a, b\} \notin \mathcal{N}$ we have $w^{\prime}(y) \neq 0$ and so $x$ and $y$ will receive different weights, thus giving an $\{a, b\}$-weight colouring of $G$. 
Theorem 3.5 Let $a, b \in \mathbb{R}$ be such that $\{a, b\} \notin \mathcal{E} \cup \mathcal{N}$. Let $G$ be a connected bipartite graph with a thread of even length $P$ and let $U$ be the internal vertices of $P$. If $G-U$ is connected then $G$ is $\{a, b\}$-weight colourable.

Proof: We may assume that $a$ is a positive odd integer and $b$ is a positive even integer. If $X \cup Y$ is the bipartition of $V(G)$ and either $|X|$ or $|Y|$ is even, then $G$ is $\{a, b\}$-weight colourable by Theorem 3.3 . Assume both parts of $G$ are of odd size. Let $x$ and $y$ be the ends of $P$. We first assume that $x$ and $y$ are distinct. By Lemma 2.8, we may assume that $P$ is a path of length either 2 or 4 .

Consider the case that $P$ is of length 2, say $P=x v y$. Let $G^{\prime}$ be the bipartite graph obtained from $G$ by deleting $v$ and adding two leaves, $v_{1}$ adjacent to $x$ and $v_{2}$ adjacent to $y$. Now $G^{\prime}$ is connected and bipartite with an even side, where $v_{1}$ and $v_{2}$ both belong to the even side. Theorem 3.3 gives an $\{a, b\}$-weight colouring of $G^{\prime}$, say $w^{\prime}$, so that $v_{1}$ and $v_{2}$ are both odd vertices. Hence $x v_{1}$ and $y v_{2}$ must both receive $a$ as their weight. Let $w$ be an $\{a, b\}$-edge-weighting of $G$, where $w(x v)=w^{\prime}\left(x v_{1}\right)=a, w(y v)=$ $w^{\prime}\left(y v_{2}\right)=a$ and $w(e)=w^{\prime}(e)$ for all other edges $e \in E(G)$. If $w$ is not an $\{a, b\}$-weight colouring of $G$, then either $w(x)=2 a$ or $w(y)=2 a$. Without loss of generality, suppose $w(x)=r a+s b=2 a$ (a similar argument will hold for $y$ ). Since $w(x v)=a$ and $r$ even, we have $r \geq 2$. If $r=2$, then $s b=0$ which implies $s=0$ or equivalently $\operatorname{deg}_{G}(x)=2$, a contradiction. If $r \geq 3$, then $s b<0$ which gives $b<0$, a contradiction. Thus $w$ is an $\{a, b\}$-weight colouring of $G$.

Suppose $|P|=4$. Let $P=x v_{1} v_{2} v_{3} y$ and let $G^{\prime}=G-v_{2}$. Now $G^{\prime}$ is bipartite with an even side $X^{\prime}$, and $x, y \in X^{\prime}$. Theorem 3.3 gives an $\{a, b\}$-weight colouring of $G^{\prime}$, say $w^{\prime}$, so that $v_{1}$ and $v_{3}$ are both even vertices. Hence $x v_{1}$ and $y v_{3}$ must both receive $b$ as their weight. Let $w$ be an $\{a, b\}$-edge-weighting of $G$, where $w\left(v_{1} v_{2}\right)=w\left(v_{2} v_{3}\right)=a$ and $w(e)=w^{\prime}(e)$ for all other edges $e \in E(G)$. If $w$ is not an $\{a, b\}$-weight colouring of $G$, then either $w(x)=a+b$ or $w(y)=a+b$. Suppose $w(x)=r a+s b=a+b$. Then $(r-1) a=-(s-1) b$, and thus $r$ is odd. Again, we have that $a$ and $b$ are positive integers. Thus either $r-1<0$ or $s-1<0$. However, since $w\left(x v_{1}\right)=b$, we have $s \neq 0$, and since $r$ is odd, $r \neq 0$. Thus $w$ is an $\{a, b\}$-weight colouring of $G$.

Now, suppose $x$ and $y$ are not distinct (call this vertex $x$ ). Then $P$ is a cycle which is an end block of $G$ and $x$ is a cut vertex of $G$. Let $z_{1}$ and $z_{2}$ be the neighbours of $x$ in $P$. Since $G^{\prime}=G-U$ is a connected bipartite graph with one part having even size, then by Theorem 3.3 there is an $\{a, b\}$-weight colouring of $G^{\prime}$, say $w^{\prime}$. We give an edge weighting $w^{\prime \prime}$ of $P$ as follows:

- if $P$ has length $2(\bmod 4)$, then by Proposition 2.12 we may define an $\{a, b\}$-weight colouring of $P, w^{\prime \prime}$, so that $w^{\prime \prime}\left(z_{1}\right)=w^{\prime \prime}(x)=w^{\prime \prime}\left(z_{2}\right)=2 a$ and $P$ is properly coloured elsewhere;

- if $P$ has length $0(\bmod 4)$, then by Proposition 2.9 we may define an $\{a, b\}$-weight colouring of $P, w^{\prime \prime}$, so that $w^{\prime \prime}(x)$ is the larger of $2 a$ and $2 b$ and $P$ is properly coloured.

Let $w$ be the weighting obtained by combining $w^{\prime}$ and $w^{\prime \prime}$. Then $w(x)>w\left(z_{1}\right), w\left(z_{2}\right)$ and $x$ has the same parity under $w$ as under $w^{\prime}$. Hence the weight of $x$ is distinct from its neighbours in $G$. Since all other vertices are properly coloured by $w^{\prime}$ or $w^{\prime \prime}, w$ gives an $\{a, b\}$-weight colouring of $G$.

Theorem 3.6 If $G$ is a 2-connected round graph which is not a cycle then $G$ contains at least 2 even ears.

Proof: 
We first claim that $G$ contains no proper 2-connected subgraph which contains all even ears of $G$. Toward a contradiction suppose $H$ is a 2-connected maximal proper subgraph of $G$ that contains all even ears of $G$. There exist two vertices of $H$, say $x$ and $y$, which are connected by a path $P$ such that $H \cap P=\{x, y\}$. Since $H$ is 2-connected, there are also 2 edge disjoint paths $P^{\prime}$ and $P^{\prime \prime}$ in $H$ between $x$ and $y$. Thus $P \cup P^{\prime} \cup P^{\prime \prime}$ is a theta graph, and by Lemma $3.2 P$ must be of even length. Since $H$ already contains all even ears of $G, H^{\prime}=H \cup P$ must be a proper subgraph of $G$ but $H^{\prime}$ is also 2-connected which contradicts the maximality of $H$.

Now, if $G$ has no even ear, then any cycle of $G$ is a 2-connected subgraph containing all the even ears and this is a contradiction as $G$ is not a cycle. If $G$ has only one ear, let $T$ be the ear and let $x$ and $y$ be the two ends of $T$. Then there are 2 edge disjoint paths connecting $x$ and $y$, one of which must be edge disjoint from $T$. This path together with $T$ forms a cycle that contains all the even ears of $G$, a contradiction.

Corollary 3.7 If $G$ is a round graph and all threads of $G$ are odd, then $G$ has at least two leaves. 2 .

We are now able to prove that round graphs can be edge-weight vertex-coloured with most sets of size

Theorem 3.8 Every round graph is $\{a, b\}$-weight colourable for $\{a, b\} \notin \mathcal{E} \cup \mathcal{N}$.

Proof: Let $G$ be a round graph. Let $B$ be an end block with vertex of attachment $v$. If $B$ is isomorphic to $K_{2}$, then $G$ is a bipartite graph with a leaf and thus is $\{a, b\}$-weight colourable by Corollary 3.4 If $B$ is a cycle, then $B$ is an even thread and $G$ is $\{a, b\}$-weight colourable by Theorem 3.5 . Otherwise, if $B$ is a 2-connected graph which is not a cycle, then by Theorem 3.6, $B$ has at least two even ears and thus $B$ has at least one even ear, say $P$, which does not contain $v$ as an internal vertex. Let $U$ be the internal vertices of $P$. Since $G-U$ is connected, $G$ is $\{a, b\}$-weight colourable by Theorem 3.5

Theorem 3.8, together with Proposition 2.9 gives a class of minimal subgraphs with respect to containment which cannot be $\{a, b\}$-weight coloured for the pairs $\{a, b\}$ on which we have focused.

Corollary 3.9 Let $a$ and $b$ be real numbers such that $\{a, b\} \notin \mathcal{E} \cup \mathcal{N}$. Any graph which is not $\{a, b\}$ weight colourable must contain a cycle of length 1,2 or $3 \bmod 4$.

We end this section with the following problem.

Problem 3 Is it true that all bipartite graphs except $C_{4 k+2}$ and $\Theta_{\left(1,4 k_{1}+1,4 k_{2}+1, \ldots, 4 k_{d}+1\right)}$ are 2-weight colourable?

\section{More families of graphs with determined 2-weight colourability}

We have given a number of examples of $\{a, b\}$-weight colourable graphs for values of $a$ and $b$ subject to particular restrictions. However we have seen few examples of graphs for which $a$ and $b$ can be any distinct real numbers. We note that the Petersen graph provides such an example of a 2-weight colourable graph. One such edge-weighting is given in Figure 1. By Proposition 2.3. note that any 2-weight colouring of the Petersen graph gives a 3-colouring of it, which is also an optimal proper vertex colouring.

In the rest of this section we describe more families of 2-weight colourable graphs as well as a class of nonbipartite graphs which are $\{a, b\}$-weight colourable when $a b>0$. In particular we show that all 


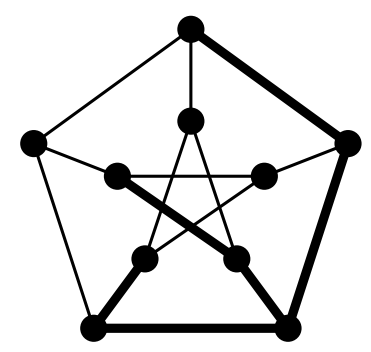

Fig. 1: An $\{a, b\}$-weight colouring of the Petersen graph. Bold edges are to receive weight $b$.

unicyclic graphs except cycles of length 1,2,3 $\bmod 4$ are 2 -weight colourable. We also provide a number of results on Cartesian products of graphs. Finally, we explore techniques for constructing graphs which do not admit $\{a, b\}$-weight colourings for any choice of $a$ and $b$.

We begin with our result on unicyclic graphs.

Theorem 4.1 Every connected unicyclic graph except $C_{2 k+1}$ and $C_{4 m+2}$ is $\{a, b\}$-weight colourable, where $a$ and $b$ are real numbers with $a b>0$.

Proof: We may assume that $0<a<b$. By contradiction, let $G$ be the smallest counterexample to our claim. Let $C$ be the only cycle of $G$. We first note that by Lemma 2.6, we may assume that every vertex of $G$ is either on $C$ or is adjacent to a vertex of $C$. We may also assume that every vertex of $G$ has degree at most 3. Next, we claim that there are at least two vertices of degree at least 3 on $C$. If not, let $v$ be the only vertex of degree at least 3 on $C$. Let $x$ and $y$ be the neighbours of $v$ on $C$. It is easy to find an edge-weighting $w$ of $C$ which yields a proper colouring on $C-v$ and $w(v) \geq w(x), w(y)$. By assigning $b$ to the other edge incident with $v$, we get an $\{a, b\}$-weight colouring of $G$, a contradiction.

Next, we claim that $G$ has at most one ear of length at least 2. If not, then we choose some maximal path of degree 3 vertices on $C, x_{1}, \ldots, x_{k}$, and remove all leaves of $G$ adjacent to those vertices. Call this subgraph $G^{\prime}$. By minimality of our choice of $G$, we can assign an $\{a, b\}$-weight colouring $w^{\prime}$ to $G^{\prime}$. Let $w$ be the weighting of $E(G)$ given by $w(e)=w^{\prime}(e)$ if $e \in E\left(G^{\prime}\right)$ and $w(e)=b$ otherwise. The only possible conflicts are between $x_{1}$ and its neighbour on $C$ which is not $x_{2}$, say $y$ (or, similarly, between $x_{k}$ and it's neighbour on $C$ which is not $\left.x_{k-1}\right)$. However, since $w\left(x_{1}\right) \geq a+b+w\left(x_{1} y\right)$ and $w(y) \leq w\left(x_{1} y\right)+b$ (similar for $\left.x_{k}\right), w$ is an $\{a, b\}$-weight colouring of $G$ which contradicts our choice of $G$.

If $G$ has exactly one ear of length at least 2, let $e=r s$ and $e^{\prime}=r^{\prime} s^{\prime}$ be the two edges that have exactly one endpoint of degree 2. Specifically, let $\operatorname{deg}(r)=\operatorname{deg}\left(r^{\prime}\right)=2, \operatorname{deg}(s)=\operatorname{deg}\left(s^{\prime}\right)=3$. Note that $r$ and $r^{\prime}$ need not be distinct, but, since there are at least 2 vertices on $C$ of degree $3, s$ and $s^{\prime}$ are distinct. We construct an $\{a, b\}$-weight colouring of $G$ based on the length of $C \bmod 4$.

- Suppose $|C|$ is odd. By Proposition 2.10, $C$ has an $\{a, b\}$-edge weighting $w^{\prime}$ which gives a proper vertex colouring except across $r s$. Let $w(e)=w^{\prime}(e)$ if $e \in E(C)$. If $w^{\prime}\left(r^{\prime}\right)-w^{\prime}\left(s^{\prime}\right)=a$, let $w(e)=b$ for all $e \in E(G) \backslash E(C)$. Otherwise, let $w(e)=a$ for all $e \in E(G) \backslash E(C)$. Clearly each leaf's neighbour has a weight strictly greater than its own. Since $w^{\prime}$ gives a proper colouring of $C$ except for $r$ and $s$, the only adjacent vertices of $G$ which might not be properly coloured are $r$ 
and $s$ or $r^{\prime}$ and $s^{\prime}$. However, our choice of weights for the leaves of $G$ guarantees that $r, s, r^{\prime}, s^{\prime}$ are properly coloured as well. Thus $w$ is an $\{a, b\}$-weight colouring of $G$.

- Suppose $|C| \equiv 0(\bmod 4)$. By Proposition 2.9, $C$ has an $\{a, b\}$-weight colouring $w^{\prime}$ such that $w(r)=2 a$ and $w(s)=a+b$. Let $w(e)=w^{\prime}(e)$ if $e \in E(C)$. If $w^{\prime}\left(r^{\prime}\right)-w^{\prime}\left(s^{\prime}\right)=a$, let $w(e)=b$ for all $e \in E(G) \backslash E(C)$. Otherwise, let $w(e)=a$ for all $e \in E(G) \backslash E(C)$. By the same argument as above, $w$ is an $\{a, b\}$-weight colouring of $G$.

- Suppose $|C| \equiv 2(\bmod 4)$. Let $t$ be the other neighbour of $s$ on $C$ and let $t^{\prime}$ be the other neighbour of $r^{\prime}$ on $C$. By Proposition 2.11 there is an $\{a, b\}$-edge weighting such that all vertices are properly coloured except $r, s$ and $t$, and such that $w\left(t^{\prime} r^{\prime}\right)=w\left(r^{\prime} s^{\prime}\right)=a$. Let $w(e)=w^{\prime}(e)$ for all $e \in$ $E(C)$. Let $f$ be the edge between $s$ and its leaf, and let $w(f)=a$. For each $e \in E(G) \backslash E(C) \backslash\{f\}$, let $w(e)=b$. The only possible improperly coloured pairs of vertices are $r$ and $s, s$ and $t$ or $r^{\prime}$ and $s^{\prime}$. However,

$$
\begin{aligned}
w(r) & =w^{\prime}(r)=w^{\prime}(s)<w(s) \\
w(s) & =w^{\prime}(s)+a=w^{\prime}(t)+a<w^{\prime}(t)+b=w(t) \\
w\left(r^{\prime}\right) & =2 a<a+2 b=w\left(s^{\prime}\right)
\end{aligned}
$$

and so $w$ is an $\{a, b\}$-weight colouring of $G$.

The only remaining case is that every vertex of $C$ has degree 3. If $|C|$ is even, assign the same weight to all the edges on the cycle and alternating weights to the leaf edges. The reader can verify that a solution for the cases when $|C|=3$ or $|C|=5$ exists. Each of these cases can be extended to larger odd cycle by making the replacement indicated in Figure 2 Note that the variables $\bar{\ell}$ and $\bar{n}$ refer to the weights different from $\ell$ and $n$, respectively.

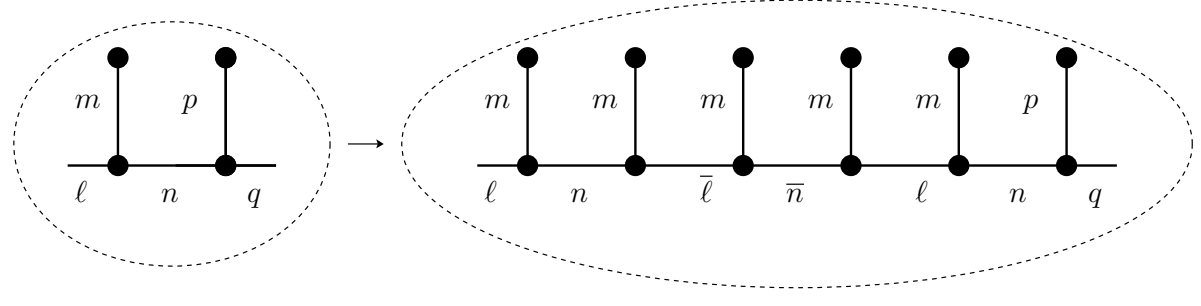

Fig. 2: Replacement operation to expand 2-weight colourings to larger cycles.

Thus, no minimal counterexample $G$ exists.

Proposition 4.2 For $n \geq 4$, the graph $K_{2} \square K_{n}$ is 2-weight colourable.

Proof: Let $K_{n}$ and $K_{n}^{\prime}$ be the two copies of the complete graph. Denote the vertices of $K_{n}$ and $K_{n}^{\prime}$, respectively by

$$
\begin{aligned}
& \left\{u_{1}, u_{2}, \ldots, u_{\lfloor n / 2\rfloor}, v_{\lfloor n / 2\rfloor}, v_{\lfloor n / 2\rfloor+1}, \ldots, v_{n-2}, v_{n-1}\right\} \\
& \left\{u_{1}^{\prime}, u_{2}^{\prime}, \ldots, u_{\lfloor n / 2\rfloor}^{\prime}, v_{\lfloor n / 2\rfloor}^{\prime}, v_{\lfloor n / 2\rfloor+1}^{\prime}, \ldots, v_{n-2}^{\prime}, v_{n-1}^{\prime}\right\}
\end{aligned}
$$


Let $p$ be a derangement (permutation with no fixed points) of $\{1,\lfloor n / 2\rfloor\}$ and $\pi$ be a derangement of $\{\lfloor n / 2\rfloor, n-1\}$. Let $u_{i}$ be adjacent to $u_{p(i)}^{\prime}$ for all $1 \leq i \leq\lfloor n / 2\rfloor$ and $v_{i}$ be adjacent to $v_{\pi(i)}^{\prime}$ for $\lfloor n / 2\rfloor \leq i \leq n-1$.

Since the graph is $n$-regular, if adjacent vertices have distinct weights then they have distinct numbers of incident edges having weight $b$. Using Lemma 2.5. we may weight the edges of $K_{n}$ and $K_{n}^{\prime}$ so that the subscript of the vertex is precisely equal to the number of edges weighted $b$ incident to that edge in $K_{n}$. Label $u_{i} u_{p(i)}^{\prime}$ with $a$ for all $1 \leq i \leq\lfloor n / 2\rfloor$ and weight $v_{i} v_{\pi(i)}^{\prime}$ with $b$ for $\lfloor n / 2\rfloor \leq i \leq n-1$. Then any two vertices that are adjacent have a distinct number of incident edges weighted $b$ and thus $K_{2} \square K_{n}$ is 2-weight colourable.

Figure 3 gives an illustration of this construction.

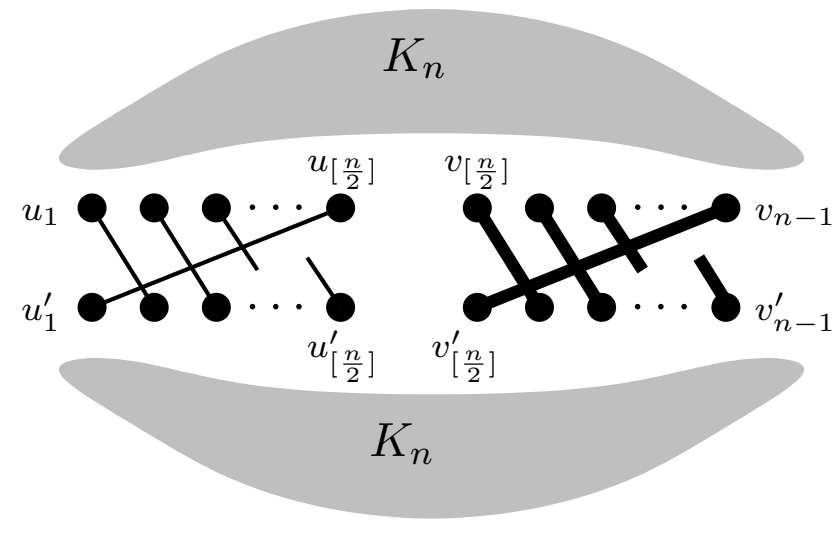

Fig. 3: An $\{a, b\}$-weight colouring of $K_{2} \square K_{n}$. Bold edges are to receive weight $b$.

Proposition 4.3 The graph $K_{2} \square C_{n}$ is 2-weight colourable if and only if $n \geq 4$ and $n \neq 5$.

Proof: If $n$ is even, then give every edge of one copy of $C_{n}$ weight $a$ and every edge of the other copy weight $b$. By alternating the weights of the images of $K_{2}$ between $a$ and $b$ along the cycles, we have the desired $\{a, b\}$-weight colouring.

An example of an $\{a, b\}$-weight colouring of $K_{2} \square C_{7}$ is given in Figure 4. It can be extended to an $\{a, b\}$-weight colouring of $K_{2} \square C_{9}$ by replacing the left subgraph in Figure 5 with the right graph. Note that the right subgraph contains the left one, and thus this operation may be repeated as many times as needed to give an $\{a, b\}$-weight colouring for any $K_{2} \square C_{2 k+1}(k \geq 3)$ The reader may verify that no $\{a, b\}$-weight colouring of $K_{2} \square C_{3}$ or $K_{2} \square C_{5}$ exists.

Theorem 4.4 Let $G$ be a graph and $H$ be a regular bipartite graph. If $G \square K_{2}$ is 2-weight colourable, then $G \square H$ is 2-weight colourable.

Proof: Let $w$ be an $\{a, b\}$-weight colouring of $G \square K_{2}$. Denote the two copies of $G$ by $G_{1}$ and $G_{2}$ and denote the vertices of $K_{2}$ by $t_{1}$ and $t_{2}$. Since $H$ is regular (say $d$-regular) and bipartite, Hall's Theorem guarantees a perfect matching $M$ of $H$. Let $X$ and $Y$ be the parts of $V(H)$. 


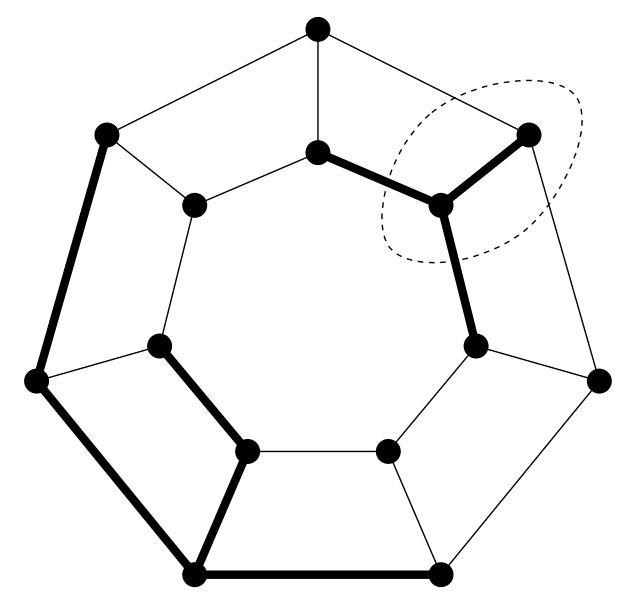

Fig. 4: An $\{a, b\}$-weight colouring of $K_{2} \square C_{7}$. Bold edges are to receive weight $b$.

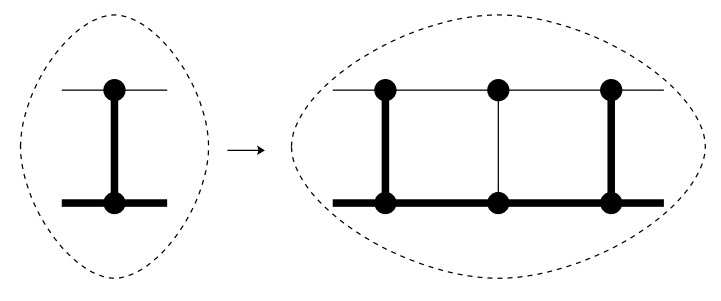

Fig. 5: Replacement operation for obtaining an $\{a, b\}$-weight colouring of $K_{2} \square C_{2 k+1}$ for $k \geq 4$.

Define an edge-weighting of $G \square H$ as follows. For each edge $e=x y \in M$ where $x \in X$ and $y \in Y$, weight the edges of the subgraph $G \square e$ by $w$ so that each vertex $\left(u_{G}, x\right) \in V(G \square H)$ has weight $w\left(u_{G}, t_{1}\right)$ and $\left(u_{G}, y\right) \in V(G \square H)$ has weight $w\left(u_{G}, t_{2}\right)$. Assign every other edge of $G \square H$ weight $a$. Call this weighting $\phi$.

We have that $\phi(u)=w\left(u_{G}, t_{1}\right)+(d-1) a$ if $u_{H} \in X$ and $\phi(u)=w\left(u_{G}, t_{2}\right)+(d-1) a$ if $u_{H} \in Y$. Two vertices are adjacent if either their $H$-coordinates agree and they are adjacent in a copy of $G$ or if their $G$-coordinates agree and they are adjacent in a copy of $H$. In the former case, their weights are distinct under $\phi$ since they are distinct under $w$. In the latter, consider two adjacent vertices $u=\left(u_{G}, u_{H}\right)$ and $u^{\prime}=\left(u_{G}, u_{H}^{\prime}\right)$ where $u_{H} \in X, u_{H}^{\prime} \in Y$. Then, $w\left(u_{G}, t_{1}\right) \neq w\left(u_{G}, t_{2}\right)$ by choice of $w$, which implies that $\phi(u) \neq \phi\left(u^{\prime}\right)$. Thus $\phi$ is an $\{a, b\}$-weight colouring of $G \square H$.

Corollary 4.5 If $G$ and $H$ are regular bipartite graphs, then the following graphs are 2-weight colourable:

(i) $K_{n} \square H$, if $n \geq 4$

(ii) $C_{n} \square H$ if $n \geq 4, n \neq 5$

(iii) $G \square H$ 
Proof: Applying Theorem 4.4 to Propositions 4.2 and 4.3 immediately gives results (i) and (ii) respectively. For (iii), since $K_{2} \square K_{2} \cong C_{4}, K_{2} \square K_{2}$ is 2-weight colourable by Proposition 2.9 By Theorem 4.4. $K_{2} \square H$ is 2-weight colourable; applying Theorem 4.4 again gives us that $G \square H$ is 2 -weight colourable.

In order to construct non-2-weight colourable graphs below, we make use of a class of "gadget" graphs. These gadgets are themselves 2-weight colourable, but they have the property that in any of their 2-weight colourings, certain edges receive a predetermined weight.

Define the graph $\widehat{K}_{n}$ to be the graph obtained from $K_{n}$ by subdividing one edge exactly once.

Proposition 4.6 For $n \geq 4$, the graph $\widehat{K}_{n}$ is 2-weight colourable. Moreover, in any 2-weight colouring of $\widehat{K}_{n}$, the edges incident to its degree 2 vertex must receive the same colour.

Proof: Let $x$ be the vertex of $\widehat{K}_{n}$ of degree 2 and let $u, v$ be its neighbours. An $\{a, b\}$-weight colouring of $\widehat{K}_{4}$ is given in Figure 6 . So assume $n \geq 5$. Let $K_{n}$ be obtained by adding the edge $u v$ to $\widehat{K}_{n}-x$. By Lemma 2.5, there exists an edge-weighting $w$ of $K_{n}$ such that all the vertices have distinct weighted degrees except for $u$ and $v$. Moreover, $w(u)=w(v)=r a+(n-1-r) b$, where $r \in\{\lfloor n / 2\rfloor,\lceil n / 2\rceil-1\}$. Assign the weight $w(u v)$ from $K_{n}$ to the edges $x u$ and $x v$ in $\widehat{K}_{n}$. Note that $w$ is an $\{a, b\}$-weight colouring as long as $w(u)=w(v) \neq w(x)$. We have $w(x) \in\{2 a, 2 b\}$. Since $a$ and $b$ may be swapped in Lemma 2.5, we assume that $w(x)=2 a$. If $w(u) \neq 2 a$, we are done. Suppose $w(u)=2 a$. We consider two cases:

- If $n$ is odd, then the edge weighting $w^{\prime}$ given by swapping every edge's weight gives $w^{\prime}(u)=$ $w(u)=2 a \neq 2 b=w^{\prime}(x)$.

- If $n$ is even then, by the construction of the weighting in Lemma 2.5 $w(u)=\frac{n}{2} a+\left(\frac{n}{2}-1\right) b$. So, $2 a=\frac{n}{2}(a+b)-b$. If the edge weighting $w^{\prime}$ given by swapping every edge's weight gives a conflict between $u$ and $x$, then $2 b=\frac{n}{2}(a+b)-a$. Together, these imply that $a=b$, a contradiction.

Thus $\widehat{K}_{n}$ admits an $\{a, b\}$-weight colouring.

To prove the second part, toward a contradiction, suppose $\widehat{K}_{n}$ is the smallest counterexample for which there exists an $\{a, b\}$-weight colouring $w$ such that $w(x u) \neq w(x v)$. By inspection, we may check that $\widehat{K}_{4}$ does not admit such edge-weighting. So assume $n \geq 5$. Note that there exists no vertex $y \neq u, v$ such that $w(y) \in\{(n-1) a,(n-1) b\}$, otherwise $\widehat{K}_{n}-y$ would be a smaller counterexample. Therefore, since $w$ induces a vertex colouring and all the weighted degrees (except for $x$ ) are of the form $r a+(n-1-r) b$ for some $0 \leq r \leq n-1$, we must have $w(u), w(v) \in\{(n-1) a,(n-1) b\}$. But then by removing $u, v$, and $x$ we get an $\{a, b\}$-weight colouring of $K_{n-2}$, a contradiction to Corollary 2.4 .

Corollary 4.7 Given a graph $G$, let $G^{\prime}$ be obtained from identifying a vertex of $G$ with the degree 2 vertex of $\widehat{K}_{n}$. Then in any 2-weight colouring of $G^{\prime}$, edges in $\hat{K}_{n}$ incident to its degree 2 vertex must receive same colour.

Proof: Since the proof of Proposition 4.6 did not depend in any way on the accumulated weight at vertex $x$, then regardless of graph joined to $K_{n}$ at $x$, the two edges incident with $x$ in $\hat{K}_{n}$ must still receive the same weight. 
An example is given on the left of Figure 6 In the case $G=K_{2}$ and $n=4$, the weight of the leaf's edge is forced to be equal to that of its incident edges; this is another useful gadget. It is shown on the right of Figure 6
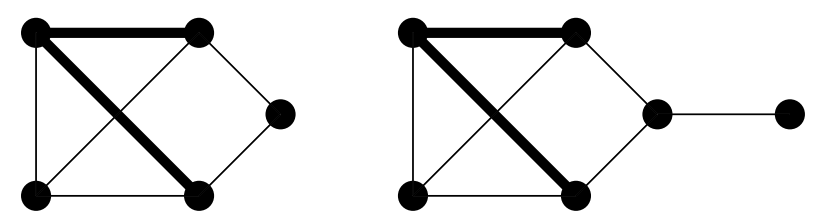

Fig. 6: The graphs $\hat{K}_{4}$ and $\hat{K}_{4}$ with a leaf are 2-weight colourable. Bold edges represent one weight-class.

We use Proposition 4.6, which established the weight colourability of $\hat{K}_{n}$, to construct the following examples of non 2-weight colourable graphs.

Example 4.8 The following graphs cannot be 2-weight coloured:

(i) Join two copies of $\hat{K}_{4}$ by an edge attached at their vertices of degree 2.

(ii) Join $2 n+1$ copies of $\hat{K}_{4}$ to a $C_{2 n+1}$ by an edge attaching the degree 2 vertex in each copy of $\hat{K}_{4}$ to a distinct cycle vertex.

To see why the graph defined in (ii), which we denote $H$, cannot be $S$-weight coloured for any set $S$ of size 2, consider the accumulated weight at one of the cycle vertices, say $v$. Since $H$ is 3-regular graph, $w(v) \in\{3 a, 2 a+b, a+2 b, 3 b\}$. If $w(v)=3 a$, then the noncycle edge, $e$, incident with $v$ must have weight $a$ and, as shown in Figure 6 so must the two edges in the copy of $\hat{K}_{4}$ joined to $v$ by an edge. Thus both endpoints of e would have weight $3 a$. A similar argument shows that $w(v) \neq 3 b$. Thus the only possible accumulated weights on cycle vertices are $2 a+b$ and $2 b+a$. Since an odd cycle cannot be properly 2-coloured, we see that $H$ cannot be 2-weight coloured.

Our next family of gadget graphs are described below.

Proposition 4.9 Let $0 \leq a \in \mathbb{Z}$ and $d \mid a$. Let $H$ be a graph and $G$ be a graph obtained from identifying a vertex $u$ of $H$ with a vertex of a $K_{n}$ (all other vertices of $H$ and $K_{n}$ being disjoint). If

$$
\operatorname{deg}_{H}(u)<\left(\frac{d}{a+d}\right)\left\lfloor\frac{n-1}{2}\right\rfloor,
$$

then $G$ is not $\{a, a+d\}$-weight colourable. Furthermore, if

$$
\operatorname{deg}_{H}(u)=\left(\frac{d}{a+d}\right)\left\lfloor\frac{n-1}{2}\right\rfloor,
$$

then in any $\{a, a+d\}$-weight colouring of $G$, all edges in $H$ incident to $u$ must receive weight $a+d$.

Proof: We first prove the statement for $d=1$. Toward a contradiction, suppose $w$ is an $\{a, a+1\}$ weighting colouring of $G$. Every vertex of $K_{n}-u$ has weighted degree $r a+(n-1-r)(a+1)=$ $(n-1)(a+1)-r$ for some $0 \leq r \leq n-1$ and both of the weights $(n-1) a$ and $(n-1)(a+1)$ cannot 
appear simultaneously on $K_{n}-u$. If $w(u)<(n-1)(a+1)$ then there are only $n-1$ colours available for the vertices of $K_{n}$, a contradiction. So $w(u) \geq(n-1)(a+1)$.

Let $\left.w\right|_{K_{n}}$ be the edge-weighting of $K_{n}$ induced by $w$. By Corollary 2.4 $K_{n}$ is not $\{a, a+1\}$-edgeweight colourable. Thus, there must be exactly two vertices of $K_{n}$ with the same weight given by $\left.w\right|_{K_{n}}$ and $u$ must be one such vertex. By Lemma 2.5, we get $\left.w\right|_{K_{n}}(u)=r a+(n-1-r)(a+1)=(n-$ 1) $(a+1)-r$, where $r \in\{\lfloor n / 2\rfloor,\lceil n / 2\rceil-1\}$. Note that $r \geq\lfloor(n-1) / 2\rfloor$. If $u$ is incident with $s$ edges of weight $a$ in $H$, then we have

$$
(n-1)(a+1) \leq w(u)=r a+(n-1-r)(a+1)+s a+\left(\operatorname{deg}_{H}(u)-s\right)(a+1),
$$

which simplifies to $\operatorname{deg}_{H}(u) \geq\left(\frac{1}{a+1}\right)(r+s)$. Hence

$$
\operatorname{deg}_{H}(u) \geq\left(\frac{1}{a+1}\right)\left(\left\lfloor\frac{n-1}{2}\right\rfloor+s\right) .
$$

This is a contradiction since $\operatorname{deg}_{H}(u)<\left(\frac{1}{a+1}\right)\left\lfloor\frac{n-1}{2}\right\rfloor$. Also, if $\operatorname{deg}_{H}(u)=\left(\frac{1}{a+1}\right)\left\lfloor\frac{n-1}{2}\right\rfloor$ then we must have $s=0$, proving the second claim.

Now, let $d$ be any positive divisor of $a$. By Proposition 2.1. $G$ has an $\{a, a+d\}$-weight colouring if and only if it has a $\left\{\frac{a}{d}, \frac{a}{d}+1\right\}$-weight colouring. If $\operatorname{deg}_{H}(u)<\left(\frac{1}{a / d+1}\right)\left\lfloor\frac{n-1}{2}\right\rfloor$ then $G$ has no $\left\{\frac{a}{d}, \frac{a}{d}+1\right\}$-weight colouring by the above argument. Hence, if $\operatorname{deg}_{H}(u)<\left(\frac{d}{a+d}\right)\left\lfloor\frac{n-1}{2}\right\rfloor$ then $G$ has no $\{a, a+d\}$-weight colouring. The second result follows similarly.

We may use Proposition 4.9 to construct many graphs which are not $\{a, a+1\}$-weight colourable and so, in particular, are not $\{1,2\}$-weight colourable. In fact, if $H$ is any graph and $u$ any vertex of $H$, then there is an $n$ large enough so that attaching $K_{n}$ to $u$ (and only to $u$ ) gives a graph which is not $\{a, a+1\}$ weight colourable. We can also use the equality condition to construct graphs with no $\{a, a+1\}$-weight colouring (for a specific $a$ ). For example, the graph obtained by joining two copies of $K_{n}, n \geq 5$, with a path of length 3 , say $e_{1}, e_{2}, e_{3}$, is not $\{a, a+1\}$-weight colourable for $a=\left\lfloor\frac{n-1}{2}\right\rfloor-1$ since the weights of $e_{1}$ and $e_{3}$ are forced to be $a+1$, and thus the ends of $e_{2}$ will receive the same weight.

We finish this section with a problem whose solution would be useful in the study of $\{1,2,3\}$-weight colourable graphs.

Problem 4 Does there exist a graph which is either

- uniquely $\{1,2,3\}$-weight colourable (up to isomorphism), or

- $\{1,2,3\}$-weight colourable where any such colouring forces certain edges of $G$ to receive a particular weight?

Moreover, does there exist a graph with either of these properties which maintains that property when it is attached in some way to another graph? 


\section{References}

$\left[\mathrm{ABDM}^{+}\right.$07] Louigi Addario-Berry, Ketan Dalal, Colin McDiarmid, Bruce A. Reed, and Andrew Thomason. Vertex-colouring edge-weightings. Combinatorica, 27(1):1-12, 2007.

[ABDR08] L. Addario-Berry, K. Dalal, and B. A. Reed. Degree constrained subgraphs. Discrete Appl. Math., 156(7):1168-1174, 2008.

[BM08] Adrian Bondy and U.S.R. Murty. “Graph Theory” Springer, 2008.

[CLWY10] Gerard Chang, Changhong Lu, Jiaojiao Wu, and Qinglin Yu Vertex-coloring edgeweightings of graphs. Taiwanese J. Math., 15(4):1807-1813, 2011

[KKP09] Maciej Kalkowski, Michal Karonski, and Florian Pfender. Vertex-coloring edgeweightings: Towards the 1-2-3-conjecture. J. Combin. Theory Ser. B, 100(3):347-349, 2010 .

[KŁT04] Michał Karoński, Tomasz Łuczak, and Andrew Thomason. Edge weights and vertex colours. J. Combin. Theory Ser. B, 91(1):151-157, 2004.

[LYZ10] Hongliang Lu, Qinglin Yu and Cun-Quan Zhang. Vertex-Coloring 2-Edge-Weightings of Graphs. European J. Combin., 32(1):21-27, 2011.

[WY08] Tao Wang and Qinglin Yu. On vertex-coloring 13-edge-weighting. Front. Math. China, 3(4):581-587, 2008. 